\title{
Thérapie génique des surdités humaines
}

\section{Défis et promesses}

> Durant les vingt dernières années, des progrès considérables ont été accomplis dans la compréhension de la pathogénie des diverses formes de surdités, congénitales ou acquises. L'identification de gènes responsables de surdité chez l'homme, l'ingénierie et la caractérisation fonctionnelle de modèles murins de certaines formes de surdité humaine ont également fait progresser la physiologie moléculaire des cellules sensorielles auditives. Ces avancées ont ouvert la voie au développement de nouvelles stratégies thérapeutiques, alternatives aux prothèses conventionnelles (amplificateurs du son) ou aux implants cochléaires permettant d'améliorer la fonction auditive. Dans cette revue, nous présentons d'abord les progrès accomplis sur le chemin de la thérapie génique des surdités au cours de la dernière décennie. Nous discutons ensuite le potentiel de la thérapie génique pour traiter les surdités acquises ou héréditaires, ainsi que les principaux obstacles qui doivent être surmontés avant qu'une application clinique puisse être envisagée. <

La surdité est le déficit sensoriel le plus fréquent chez l'homme. En France, un enfant sur 700 environ naît avec une surdité sévère ou profonde, et un enfant sur 1000 deviendra malentendant avant l'âge adulte. Aujourd'hui, on estime qu'environ $80 \%$ des cas de surdité neurosensorielle ont une cause génétique, et $20 \%$ une cause environnementale. Parmi les surdités d'origine génétique, 30 \% sont associées à d'autres symptômes (on parle alors de surdité syndromique), mais le plus souvent (70\% des cas), la surdité est isolée. Par convention, les formes isolées de surdité héréditaire sont classées selon leur mode de transmission [1] (Tableau I). Dans $85 \%$ des cas, la surdité se transmet selon un mode autosomique récessif (les surdités portent alors le préfixe DFNB), dans 10 à $15 \%$ des cas, selon un mode autosomique dominant (DFNA) et dans $1 \%$ des cas environ, selon un mode

\section{Anaïs Meyer ${ }^{1,2}$, Christine Petit ${ }^{1,2,3,4}$, Saaid Safieddine $e^{1,2,3,5}$}

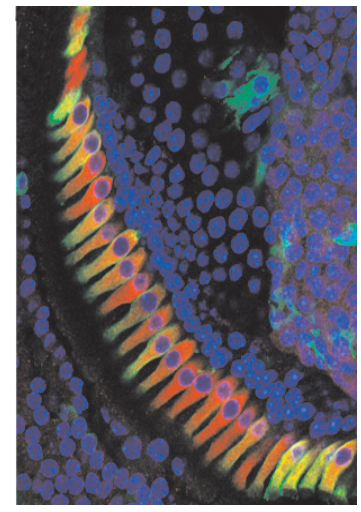

\author{
${ }^{1}$ Institut Pasteur, \\ unité de génétique et physiologie \\ de l'audition, \\ 25 , rue du Docteur Roux, \\ 75724 Paris Cedex 15, France ; \\ ${ }^{2}$ Inserm UMRS 1120, 75015 Paris, \\ France ; \\ ${ }^{3}$ Université Pierre et Marie Curie \\ (Paris VI), 75005 Paris, France; \\ ${ }^{4}$ Collège de France, \\ 75231 Paris Cedex 05, France ; \\ ${ }^{5}$ Centre national de la recherche \\ scientifique (CNRS), Paris,
}

récessif lié au chromosome $X$ (DFN). France.

Des mutations du génome mitochonssafiedd@pasteur.fr drial sont également responsables de certaines formes de surdité (Tableau I). En outre, la surdité résultant de l'exposition des adolescents à des sons trop intenses (musique amplifiée notamment) est en passe de devenir un problème médical majeur, car la perte auditive concerne des individus de plus en plus jeunes, en particulier chez les 18-30 ans (3 000 à 4000 surdités par an). Récemment, l'Organisation mondiale de la santé (OMS) a estimé qu'environ 280 millions de personnes dans le monde ont une baisse de l'acuité auditive. Ce nombre pourrait atteindre 700 millions d'ici à 2015 , et 900 millions d'ici à 2025. Bien que la connaissance des causes des surdités congénitales ou acquises ait considérablement progressé au cours des 20 dernières années, les traitements ont relativement peu évolué [2] et, à ce jour, il n'existe pas encore de traitement curatif de la surdité. Dans le cas des surdités légères ou modérées, on a généralement recours à l'amplification du son grâce à l'utilisation de prothèses auditives dites conventionnelles. Pour les surdités sévères ou profondes bilatérales, la pose chirurgicale d'un implant cochléaire, appareil qui court-circuite les cellules sensorielles auditives (cellules ciliées) non fonctionnelles et permet de stimuler directement les neurones auditifs primaires, est souvent proposée. Cependant, même si les implants cochléaires, dont les performances techniques continuent à s'améliorer, représentent une avancée significative dans le traitement des pertes auditives majeures, les patients implantés se plaignent des mauvais résultats dans un environnement bruyant [3].

Les récents succès de la thérapie génique dans le traitement de certaines formes de cécité ont fait naître l'espoir qu'une telle stratégie puisse également constituer une alternative thérapeutique pour restau- 


\begin{tabular}{|c|c|c|c|}
\hline \multicolumn{4}{|c|}{ A. Formes de surdité à transmission autosomique récessive } \\
\hline Surdités & Gènes & Protéines & $\begin{array}{l}N^{\circ} \\
\text { OMIM }\end{array}$ \\
\hline DFNBi & VEZT & Vézatine & - \\
\hline DFNBl & GJB2 & Connexine 26 & 604418 \\
\hline DFNB2 & MY07A & Myosine VIIA & 276903 \\
\hline DFNBla & GJB6 & Connexine 30 & 604418 \\
\hline DFNB2b & GJB3 & Connexine 31 & 604418 \\
\hline DFNB3 & MY015 & Myosine XV & 602666 \\
\hline DFNB4 & PDS/SLC26A4 & Pendrine & 605646 \\
\hline DFNB6 & $T M I \varepsilon$ & $\begin{array}{l}\text { Transmembrane inner ear } \\
\text { expressed }\end{array}$ & 607237 \\
\hline DFNB7/DFNB11 & $T M C 1$ & Canal sodique & 606706 \\
\hline DFNB8/10 & TMPRSS3 & Protéase & 605511 \\
\hline DFNB9 & OTOF & Otoferline & 603681 \\
\hline DFNB12 & $\mathrm{CDH} 23$ & Cadhérine 23 & 602092 \\
\hline $\begin{array}{l}\text { DFNB15/DFNB72/ } \\
\text { DFNB95 }\end{array}$ & GIPC3 & Protéine GIPC & 608792 \\
\hline DFNBI6 & STRC & Stéréociline & 606440 \\
\hline DFNB18 & USHIC & Harmonine & 605242 \\
\hline DFNB21 & TECTA & Alpha-tectorine & 602574 \\
\hline DFNB22 & Otoancorin & Otoancorine & 607038 \\
\hline DFNB23 & & Protocadhérine 15 & 605514 \\
\hline DFNB24 & $R D X$ & Radixine & 179410 \\
\hline DFNB25 & GRXCRI & Glutaredoxin cys-rich 1 & 613283 \\
\hline DFNB28 & TRIOBP & Trio/F-actin-binding protein & 609761 \\
\hline DFNB29 & CLD14 & Claudine 14 & 605608 \\
\hline DFNB30 & MY03А & Myosine IIIA & 606808 \\
\hline DFNB31 & WHRN & Whirline & 607928 \\
\hline DFNB35 & $\varepsilon S R R B$ & estrogen-related receptor-alpha & 602167 \\
\hline DFNB36 & $\varepsilon S P$ & Espine & 606351 \\
\hline DFNB37 & My06 & Myosine VI & 600970 \\
\hline DFNB39 & $H G F$ & Hepatocyte growth factor & 142409 \\
\hline DFNB42 & ILDRI & Immunoglobulin-like receptor & 609739 \\
\hline DFNB48 & CIB2 & $\begin{array}{l}\text { Calcium-and integrin-binding } \\
\text { protein } 2\end{array}$ & 605564 \\
\hline DFNB49 & TRIC & Tricelluline & 610572 \\
\hline DFNB53 & COLIIA2 & Collagène XIA2 & 120290 \\
\hline DFNB59 & PJVK & Pejvakine & 610219 \\
\hline DFNA61 & SLC26A5 & Prestine & 604943 \\
\hline DFNB63 & LRTOMT & Méthyl transférase & 612414 \\
\hline DFNB66/67 & TMHS & $\begin{array}{l}\text { Tetraspan membrane protein of } \\
\text { hair cell stereocilia }\end{array}$ & 609427 \\
\hline DFNB74 & MSRB3 & $\begin{array}{l}\text { Méthionine sulfoxide réductase } \\
\text { b3 }\end{array}$ & 613719 \\
\hline DFNB77 & LOXHDI & $\begin{array}{l}\text { Lipoxygenase homology domain- } \\
\text { containing } 1\end{array}$ & 613072 \\
\hline DFNB79 & TPRN & Taperine & 613354 \\
\hline DFNB82 & GPSM2 & $G$ protein signaling modulator 2 & 609245 \\
\hline DFNB84 & PTPRP & $\begin{array}{l}\text { Protein-tyrosine phosphatase, } \\
\text { receptor-type }\end{array}$ & 603317 \\
\hline DFNB91 & SERPINB6 & Intracellular protease inhibitor & 173321 \\
\hline
\end{tabular}

\begin{tabular}{|c|c|c|c|}
\hline \multicolumn{4}{|c|}{ B. Formes de surdité à transmission autosomique dominante } \\
\hline Surdités & Gènes & Protéines & $\mathbf{N}^{\circ} \mathrm{OMIM}$ \\
\hline DFNAi & MyolC & Myosine Ic & 606538 \\
\hline DFNAl & DIAPHI & Diaphanous 1 & 602121 \\
\hline DFNA2 & KCNO4 & Canal potassique & 603537 \\
\hline DFNA3 & GJB2 & Connexine 26 & 121011 \\
\hline DFNA2b & GJB3 & Connexine 31 & 604418 \\
\hline DFNA3b & GJB6 & Connexine 30 & 604418 \\
\hline DFNA4 & MYH14 & Myosine IIC & 608568 \\
\hline DFNA5 & DFNA5 & ? & 608798 \\
\hline DFNA6/14/38 & WFS 1 & Wolframine & 606201 \\
\hline DFNA8/DFNA12 & TECTA & Alpha-tectorine & 60257 \\
\hline DFNA9 & $\mathrm{COCH}$ & Cochline & 603196 \\
\hline DFNA10 & $\varepsilon$ EA $4_{4}$ & $\begin{array}{l}\text { Facteur de } \\
\text { transcription }\end{array}$ & 603550 \\
\hline DFNAIl & MY07A & Myosine VIla & 276903 \\
\hline DFNA13 & COLIIA2 & Collagène Xla2 & 120290 \\
\hline DFNA15 & POU4F3 & $\begin{array}{l}\text { Facteur de } \\
\text { transcription }\end{array}$ & 602460 \\
\hline DFNA17 & Муня & $\begin{array}{l}\text { Myosin heavy } \\
\text { chain } 9\end{array}$ & 160775 \\
\hline DFNA20/26 & ACTGI & Gamma-actine & 102560 \\
\hline DFNA22 & MY06 & Myosine VI & 600970 \\
\hline DFNA25 & SLC7A4 & $\begin{array}{l}\text { Transporteur } \\
\text { du glutamate }\end{array}$ & 607557 \\
\hline DFNA28 & $\begin{array}{l}\text { GRHL2, } \\
\text { TFCP2L3 }\end{array}$ & $\begin{array}{l}\text { Facteur } \\
\text { de transcription }\end{array}$ & 608576 \\
\hline DFNA36 & $T M C$ & Canal sodique & 606706 \\
\hline DFNA44 & CCDC50 & Contactine & 611051 \\
\hline DFNA48 & MY01A & Myosine la & 601478 \\
\hline DFNA50 & MITN96 & Micro-ARN & 611606 \\
\hline DFNA51 & TJP2 & $\begin{array}{l}\text { Protéine des } \\
\text { jonctions serrées }\end{array}$ & 607709 \\
\hline DFNAi & CRYM & Micro-cristalline & 123740 \\
\hline DFNA64 & $\begin{array}{l}\text { SMACI } \\
\text { DIABLO }\end{array}$ & $\begin{array}{l}\text { Activateur } \\
\text { de caspase }\end{array}$ & 605219 \\
\hline
\end{tabular}

\begin{tabular}{lcc}
\hline C. Formes de surdité liées au chromosome $\mathbf{X}$ & \\
\hline Surdités & Gènes & N OMIM $^{\circ}$ \\
\hline DFN2 & PRPS1 & 311850 \\
\hline DFN3 & POU3F4 & 300039 \\
\hline DFN6 & SMPX & 300226 \\
\hline
\end{tabular}

\begin{tabular}{lcc}
\hline D. Formes de surdité mitochondriales & \\
\hline Surdités & Gènes & N OMIM $^{\circ}$ OM \\
\hline MTRNR & $12 S$ & 561000 \\
\hline MTTS1 & tRNA Ser & 590080 \\
\hline
\end{tabular}

Tableau I. Gènes responsables de formes de surdité à transmission autosomique récessive (A), autosomique dominante (B), liée au chromosome $X$ (C), et mitochondriale (D). Les formes de surdité dont les cibles primaires sont les cellules sensorielles auditives sont indiquées en rouge; celles dont les cibles primaires sont les cellules de soutien sont indiquées en bleu. Les autres formes de surdité, dont les cibles cellulaires sont différentes ou inconnues, sont indiquées en noir. 
rer l'audition chez les malentendants [4]. La thérapie génique repose sur le transfert de matériel génétique, un transgène, dans les cellules déficientes, pour obtenir un effet thérapeutique transitoire ou permanent. Une telle approche offre la possibilité de manipuler les gènes dans les cellules cibles, soit en empêchant l'expression d'un allèle muté qui exerce un effet dominant négatif, soit en rétablissant l'expression d'un gène défectueux. Elle pourrait être également utilisée pour atténuer les effets délétères des traumatismes acoustiques, voire pour la survie ou la réparation de cellules endommagées ou défectueuses dans l'oreille interne [31]. La cochlée se prête particulièrement bien à la thérapie génique pour deux raisons principales : (1) il s'agit d'un organe relativement bien isolé du reste de l'organisme, ce qui limite la diffusion systémique de l'agent thérapeutique administré (virus, nucléotides) ; (2) la cochlée est remplie de liquides (endolymphe et périlymphe), ce qui favorise la dissémination locale des agents thérapeutiques à un grand nombre de cellules cibles (Figure 1). Actuellement, les recherches sont centrées sur les techniques à utiliser pour une meilleure efficacité de transfert des gènes aux cellules de la cochlée, et des applications spécifiques, telles que la régénération et la protection des cellules ciliées. Bien que la petite taille de sa cochlée complique la chirurgie et même l'obtention d'explants de l'organe de Corti à des fins expérimentales, la souris est actuellement l'animal de choix pour explorer les possibilités de la thérapie génique des surdités humaines. En effet, le génome de souris est entièrement séquencé, et les modèles murins naturels ou obtenus par ingénierie génétique reproduisent en général fidèlement les formes de surdité humaines correspondantes [5], ce qui a permis dans la plupart des cas de préciser le rôle des gènes de surdité identifiés [6]. De plus, la durée de vie de la souris permet à la fois d'évaluer le rôle joué par l'environnement, notamment sonore, dans l'évolution de la surdité, et d'apprécier l'efficacité d'un traitement à long terme.

\section{Stratégies de transfert de gènes pour une thérapie applicable à l'oreille interne}

Dans la thérapie génique, un gène est transféré à des cellules déficientes par l'intermédiaire de transporteurs viraux ou de cargos de membranes artificielles capables d'atteindre et de pénétrer dans la cellule cible. Le vecteur est choisi en fonction de l'objectif thérapeutique recherché, des cellules à atteindre, et du gène à transférer. Par ailleurs, l'utilisation d'un promoteur spécifique ou de vecteurs viraux particuliers permet de contrôler l'expression spatio-temporelle du gène transféré.

À ce jour, plusieurs types de vecteurs ont été utilisés comme moyen de transfert d'agents thérapeutiques dans la cochlée. Les vecteurs non viraux, tels que des plasmides seuls ou enrobés dans des composés lipidiques, ont l'avantage de présenter une toxicité faible et d'entrầner une inflammation réduite par rapport aux vecteurs viraux [7-9]. Malheureusement, ces vecteurs ont montré une efficacité de transfert limitée et un faible taux d'expression du transgène, ce qui limite leur utilité $[10,11]$. Des études plus récentes ont porté sur l'utilisation de vecteurs viraux dépourvus des séquences génomiques responsables de leur réplication, afin de cibler l'expression du transgène dans un type particulier de cellules cochléaires [12-14]. Différents vecteurs viraux ont été testés dans la cochlée : adénovirus [11], virus adéno-associés (adeno-associated virus, AAV) [7], lentivirus [7], virus herpes simplex $[15,16]$, et virus de la vaccine [16]. Le vecteur viral qui a rapidement retenu l'attention pour le transfert de gènes dans l'oreille interne est I'AAV, du fait de la simplicité de son génome et des nombreux sérotypes et variants génomiques disponibles. Ce vecteur, non pathogène et peu immunogène, permet une expression du transgène stable dans le temps. II est capable d'infecter des cellules qui ne se divisent plus (c'est le cas des cellules sensorielles de la cochlée). II persiste de façon prolongée sous forme épisomale (extrachromosomique) et a un faible risque d'intégration dans le génome [17]. Enfin, son utilisation a été récemment approuvée par l'Agence du médicament européen (EMA) pour le traitement d'une maladie génétique rare, le déficit en lipoprotéine lipase ${ }^{1}$. À ce jour, 12 sérotypes d'AAV ont été décrits et classés (AAVl à -12), et plus de 100 variants ont été isolés [18]. Chaque sérotype possède un tropisme préférentiel, offrant ainsi la possibilité d'un meilleur ciblage cellulaire du transgène. Une étude récente a montré que les sérotypes AAV1, $-2,-5$, -6 , et -8 transduisent avec une efficacité variable les cellules ciliées, les cellules de soutien et les neurones cochléaires [1214]. Toutefois, compte tenu de la taille de la capside de l'AAV (24 nm de diamètre), le transgène (cassette d'expression) ne doit pas dépasser 4,7 kb [19], ce qui limite le nombre de gènes pouvant être intégrés dans ce vecteur. Cependant, de nouvelles techniques et de nouveaux concepts sont en cours de développement afin d'augmenter la capacité d'encapsidation de l'AAV [20]. Des virus hybrides ou pseudotypés, où le génome et la capside sont de sérotypes différents, ont été également construits pour augmenter l'efficacité de la transfection [21] (Figure 2).

Des avancées significatives ont été obtenues concernant la voie d'administration dans la cochlée. L'expression du transgène a été détectée lorsqu'on applique une éponge de gélatine résorbable imbibée de la préparation virale sur la membrane de la fenêtre ronde partiellement perméabilisée par la collagénase [22]. Une autre étude a montré que le traitement de la membrane de la fenêtre ronde par l'acide hyaluronique augmente la proportion des cellules ciliées transfectées [23]. Néanmoins, pour un

${ }^{1}$ http://www.ema.europa.eu/ema/index.jsp?curl=pages/news_and_events/ news/2012/07/news_detail_001574.jsp\&mid=WC0b01ac058004d5cl. 


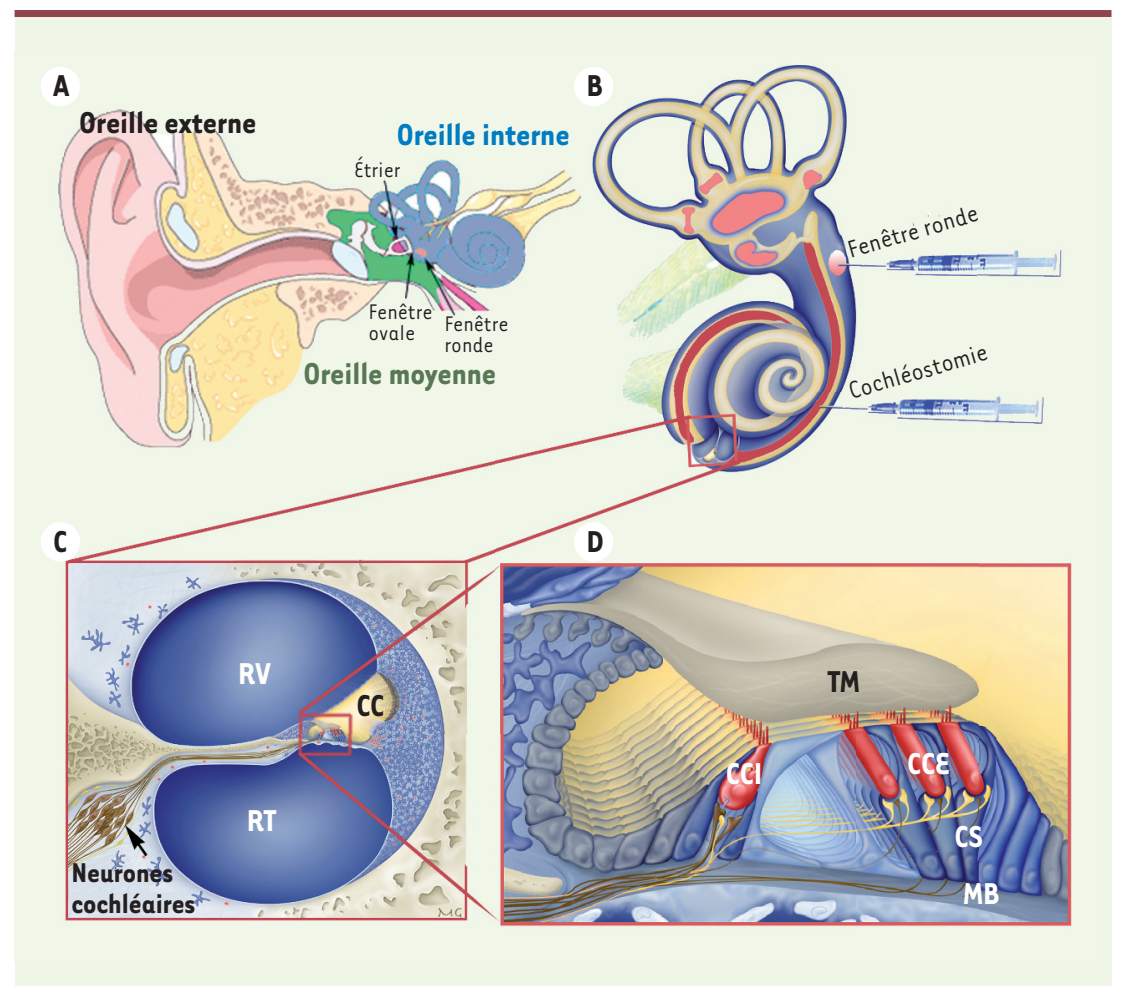

Figure 1. Représentation schématique de l'oreille humaine. A. Représentation schématique de l'oreille externe, de l'oreille moyenne et de l'oreille interne, où se trouve la cochlée (organe de l'audition). La cochlée communique avec l'oreille moyenne par deux orifices ou fenêtres, fermés chacun par une membrane : la fenêtre ronde et la fenêtre ovale, dont la membrane est en contact avec l'étrier (le dernier des trois osselets de l'oreille moyenne). B. Représentation schématique de l'oreille interne. C. Coupe transversale de la cochlée. D. Vue agrandie de l'épithélium sensoriel auditif (organe de Corti). Le canal cochléaire (CC) est rempli d'endolymphe et contient l'organe de Corti. II est séparé de la rampe vestibulaire (RV) par la membrane de Reissner, et de la rampe tympanique (RT) par la membrane basilaire (MB). L'organe de Corti comprend deux types de cellules sensorielles réparties en une rangée de cellules ciliées internes (CCI), connectées aux neurones auditifs primaires, et trois rangées de cellules ciliées externes (CCE), ainsi que des cellules de soutien (CS). L'injection, après cochléostomie ou à travers la membrane de la fenêtre ronde (B) est la technique la plus utilisée pour le transfert de gène dans l'oreille interne. TM : membrane tectoriale.

meilleur contrôle de l'expression spatio-temporelle et de la quantité d'agent thérapeutique transférée, une injection directe dans la cochlée reste l'approche la plus efficace. Nous avons observé que la microinjection intracochléaire d'AAV à travers la membrane de la fenêtre ronde de souriceaux âgés de deux jours n'affecte pas la morphologie de la cochlée (Meyer et al., résultats non publiés). Contrairement à ce qui a été décrit chez les souris injectées au stade adulte [12], les seuils auditifs enregistrés au niveau de l'oreille au-delà de la deuxième semaine postopératoire chez les animaux injectés au stade précoce sont comparables à ceux de I'oreille témoin, suggérant que la chirurgie et l'injection du vecteur AAV n'interfèrent guère avec le processus de maturation du système auditif et la fonction cochléaire. Ces résultats sont très encourageants pour développer des approches thérapeutiques applicables en clinique, avec une bonne préservation de la fonction auditive. $\varepsilon n$ effet, cette approche pourrait être une alternative à la cochléostomie (ouverture de la cochlée au niveau de la paroi latérale de la rampe tympanique), plus difficile à réaliser et moins attrayante, car elle peut provoquer des lésions de la cochlée avec une perte significative de cellules sensorielles auditives.

\section{Thérapie génique cochléaire}

Depuis une quinzaine d'années, deux grandes voies de recherche ont été développées pour tenter de restaurer l'audition par thérapie génique dans le cadre des maladies de l'oreille interne comportant des cellules ciliées défectueuses : la régénération des cellules ciliées pour les surdités acquises, et l'introduction thérapeutique d'un gène fonctionnel pour corriger les surdités génétiques.

\section{La régénération des cellules ciliées}

À la naissance, nous possédons un capital d'environ 30000 cellules sensorielles auditives, sans aucune capacité de régénération spontanée [32]. Ainsi, les pertes auditives les plus fréquentes, qui sont dues à une dégénérescence des cellules sensorielles, sont irréversibles. Une voie thérapeutique de réparation cellulaire par transfert de gènes est devenue envisageable grâce à une meilleure connaissance des gènes contrôlant le développement et la régénération des cellules ciliées. Le gène Atohl (atonal homolog 1) (Mathl chez la souris) a été l'une des premières cibles des stratégies de régénération de ces cellules [24]. Ce gène code pour un facteur de transcription qui induit la différenciation de cellules ciliées à partir des cellules de soutien de l'organe de Corti [6]. Il a été montré que l'expression du transgène Mathl véhiculé par un adénovirus induisait une régénération des cellules ciliées par transdifférenciation des cellules de soutien. 


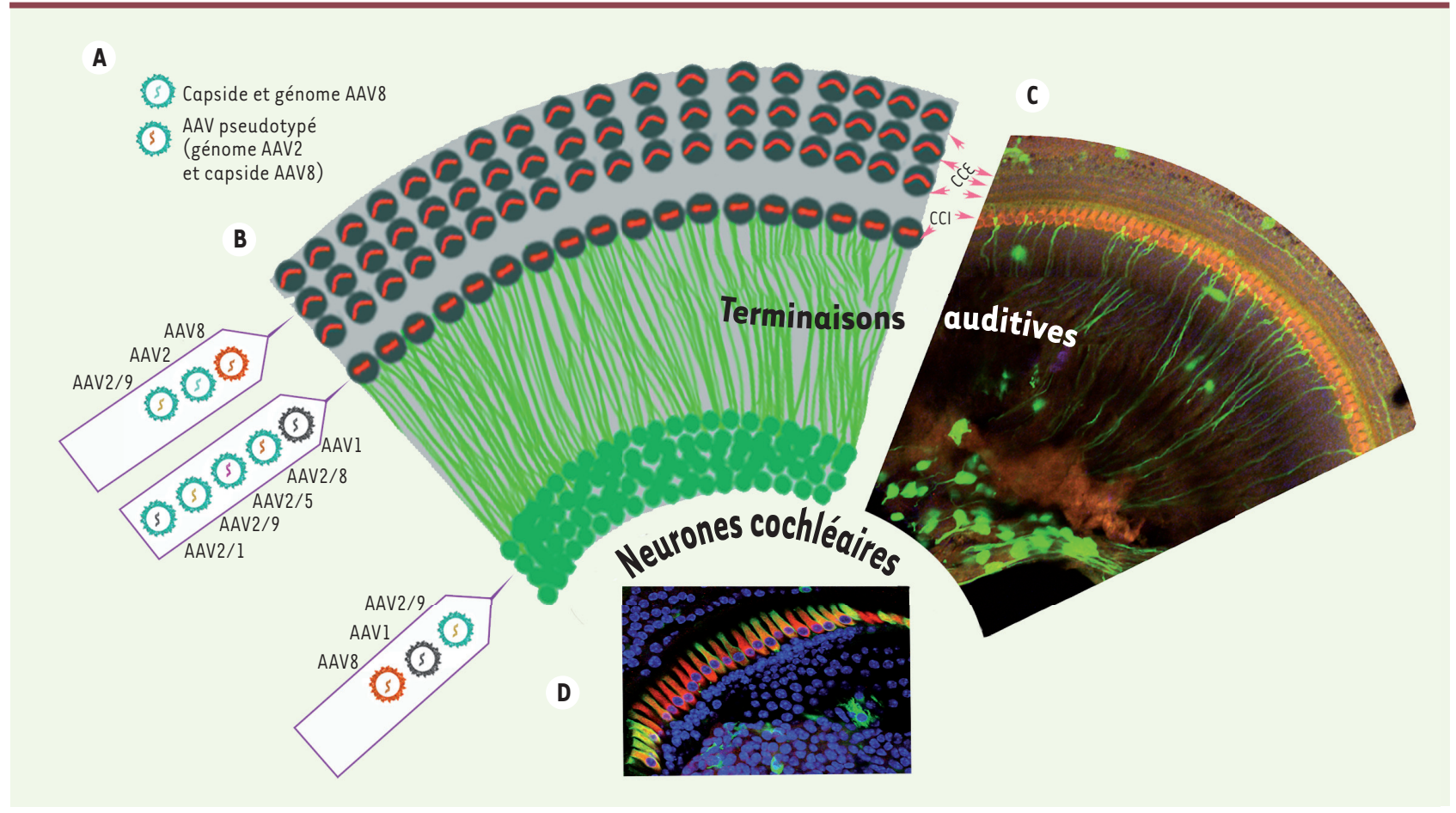

Figure 2. Transfert de gène dans l'oreille interne. A. Sérotypes d'AAV les plus couramment utilisés pour le transfert de gène dans l'oreille interne selon leur tropisme cellulaire (CCE, CCI ou neurones auditifs primaires). Les vecteurs AAV2/1, AAV2/5, AAV2/8, et AAV2/9 sont appelés «pseudotypés », car leur génome est encapsidé dans une capside d'AAV2. De tels vecteurs ont permis d'améliorer la proportion de cellules transfectées. B. Représentation schématique de l'organisation des connexions entre les neurones auditifs primaires (neurones cochléaires) et les cellules sensorielles. Les projections des neurones cochléaires (en vert) pénètrent dans le canal cochléaire pour atteindre l'organe de Corti, qui abrite les cellules ciliées (en gris foncé). C-D. Images en microscopie confocale de préparations d'organes de Corti. Ils sont doublement marqués, pour l'otoferline (en rouge), protéine produite par les cellules ciliées internes, et pour la GFP (en vert), produite par les cellules transfectées, une semaine après injection dans la cochlée, à travers la membrane de la fenêtre ronde, d'une préparation de vecteurs viraux contenant un gène rapporteur codant pour la GFP. AAV8 et AAVl transfectent de manière prédominante les neurones cochléaires (C) et les cellules ciliées internes (D), respectivement.

Cette approche a permis une récupération partielle de l'audition chez des animaux (souris et cobayes) rendus sourds [10]. La thérapie par régénération cellulaire fait l'objet de nombreux travaux, car elle est potentiellement applicable à la plupart des formes de perte auditive, notamment à la presbyacousie, ainsi qu'aux surdités consécutives à un traumatisme acoustique, une infection, ou un traitement médicamenteux ototoxique (aminoglycosides, cisplatine) [31]. Cependant, cette approche n'est en principe pas applicable aux surdités génétiques, car les nouvelles cellules sensorielles induites par Mathl subiront encore l'effet de la mutation génétique qui a conduit à leur dégénérescence. Ainsi, le remplacement du gène défectueux est nécessaire pour corriger les surdités héréditaires. Mais le traitement des surdités génétiques par thérapie génique comporte une difficulté supplémentaire. Contrairement à la régénération des cellules ciliées, pour laquelle une expression du gène approprié limitée dans le temps suffirait, l'expression durable du transgène est le plus souvent nécessaire pour corriger les surdités génétiques. C'est probablement ce qui explique le peu de résultats convaincants obtenus à ce jour par cette approche.

\section{Les surdités génétiques}

La stratégie thérapeutique peut être différente selon la forme de surdité à traiter. Les surdités transmises sur le mode autosomique dominant (environ $15 \%$ des cas) sont généralement progressives, et la pathogénie de ces surdités relève souvent d'un mécanisme dominant négatif, plutôt que d'une haplo-insuffisance. Dans ce cas, l'objectif thérapeutique est d'arrêter la progression de la surdité en inhibant l'expression de l'allèle muté sans affecter celle de l'allèle sain. Un moyen de réaliser cette inhibition sélective de l'allèle muté est d'utiliser l'interférence ARN (ARNi). Deux types de petites molécules d'ARN double brin sont utilisés pour l'interférence ARN : les petits ARNi (siARN, small interfering ARN) et les micro-ARN (miARN). Les siARN se lient à des séquences complémentaires sur I'ARN messager cible, ce qui entraîne généralement sa dégradation ou une répression de sa traduction en protéine [25]. Les miARN interfèrent avec l'expression 
de gènes spécifiques en se liant à la séquence nucléotidique complémentaire [26].

À ce jour, il existe peu de publications concernant l'utilisation de l'interférence ARN pour la thérapie des surdités. L'un des résultats les plus encourageants a été obtenu en utilisant une souris défectueuse pour le gène codant pour la connexine 26 , modèle de la forme DFNA3 de surdité autosomique dominante progressive humaine. Une éponge de gélatine résorbable imbibée de liposomes enrobant l'ARNi spécifique de la connexine 26 a été placée contre la membrane de la fenêtre ronde. Cela a permis de réduire de 70 \% l'expression de l'allèle muté et de stopper la progression de la perte auditive, sans diminution significative de l'expression de l'allèle sauvage [27]. Cela démontre que l'interférence ARN peut être utilisée in vivo avec une spécificité et une efficacité satisfaisantes pour prévenir la perte d'audition d'une surdité génétique par effet dominant négatif. Une étude récente, dans un modèle murin de la surdité autosomique dominante progressive DFNA15 par mutation du gène POU4F3 (POU class 4 homeobox 3 ), a par ailleurs montré qu'on peut empêcher la dégénérescence des neurones cochléaires par une thérapie génique via un adénovirus [28] forçant l'expression du gène codant pour le facteur de croissance BDNF (brainderived neurotrophic factor), un facteur nécessaire au développement et à la maintenance de l'innervation des cellules ciliées internes de la cochlée. Une telle approche pourrait être utilisée pour améliorer l'efficacité des implants cochléaires, dont le principe repose sur une stimulation électrique directe des fibres du nerf auditif.

Certaines surdités transmises sur le mode autosomique récessif ( $80 \%$ des cas) pourraient être traitées par transfert du gène sain correspondant au gène défectueux. Des résultats encourageants ont été obtenus chez la souris. L'audition des souris défectueuses pour la connexine 30 a été partiellement rétablie en surexprimant le gène codant pour la connexine 26 [29]. Plus récemment, une restauration de l'audition a été obtenue chez les souris homozygotes mutantes dépourvues du transporteur vésiculaire du glutamate VGLUT3. Ces souris, considérées comme un modèle de la surdité humaine autosomique dominante DFNA25, retrouvent une audition quasi normale sept jours après une seule injection intracochléaire du gène Vglut3 vectorisé dans le virus adéno-associé AAV1 [30]. Une telle approche consistant à compenser la perte de fonction du gène défectueux par transfert d'une copie saine de ce gène dans les cellules qui l'expriment, apparaît comme la plus prometteuse pour traiter efficacement et durablement certaines formes génétiques de surdité.

\section{Conclusion}

Lors de la conception d'un protocole de transfert de gène dans la cochlée, plusieurs points essentiels sont à prendre en compte : la fiabilité et la reproductibilité des résultats obtenus, la quantité et la durée de l'expression du transgène, les effets secondaires éventuels. L'existence d'une barrière hémato-cochléaire implique que le gène correcteur doit être vectorisé et injecté directement dans l'oreille interne. L'expression efficace et durable du transgène dans les cellules ciblées dépend ensuite, en grande partie, du vecteur utilisé, d'où l'importance des recherches actuelles en vectorisation. En ce qui concerne les AAV, de nombreuses possibilités restent à explorer compte tenu du nombre de sérotypes disponibles, de la possibilité de modifier la capside virale, et des multiples voies d'injection possibles dans la cochlée. La maîtrise des différentes difficultés évoquées conditionne l'application clinique des recherches actuelles et futures sur la thérapie génique. Celle-ci pourrait alors devenir un outil très utile pour la protection, la réparation et la régénération des cellules de l'oreille interne. $\diamond$

\section{SUMMARY}

Gene therapy for human hearing loss: challenges and promises

Thanks to the advances accomplished in human genomics during the last twenty years, major progress has been made towards understanding the pathogenesis of various forms of congenital or acquired deafness. The identification of deafness genes, which are potential therapeutic targets, and generation and functional characterization of murine models for human deafness forms have advanced the knowledge of the molecular physiology of auditory sensory cells. These milestones have opened the way for the development of new therapeutic strategies, alternatives to conventional prostheses, hearing amplification for mild-to-severe hearing loss, or cochlear implantation for severe-toprofound deafness. In this review, we first summarize the progress made over the last decade in using gene therapy and antisense RNA delivery, including the development of new methods for cochlear gene transfer. We then discuss the potential of gene therapy for curing acquired or inherited deafness and the major obstacles that must be overcome before clinical application can be considered. $\diamond$

\section{REMERCIEMENTS}

Les auteurs remercient chaleureusement le Dr Jean-Pierre Hardelin pour la relecture de cet article et la pertinence de ses remarques.

\section{LIENS D'INTÉRÊT}

Les auteurs déclarent n'avoir aucun lien d'intérêt concernant les données publiées dans cet article.

\section{RÉFÉRENCES}

1. Hardelin JP, Denoyelle F, Levilliers J, et al. Les surdités héréditaires : génétique moléculaire. Med Sci (Paris) $2004 ; 20: 311-6$.

2. Di Domenico M, Ricciardi C, Martone T, et al. Towards gene therapy for deafness. J Cell Physiol $2011 ; 226: 2494-9$.

3. Kral A, O'Donoghue GM. Profound deafness in childhood. N Engl J Med 2010 ; $363: 1438-50$. 


\section{RÉFÉRENCES}

4. Wojno AP, Pierce EA, Bennett J. Seeing the light. Sci Transl Med 2013 ; 5 : 175fs8.

5. Leibovici M, Safieddine S, Petit C. Mouse models for human hereditary deafness. Curr Top Dev Biol $2008 ; 84: 385-429$.

6. Bermingham NA, Hassan BA, Price SD, et al. Mathl: an essential gene for the generation of inner ear hair cells. Science $1999 ; 284: 1837-41$.

7. Bedrosian JC, Gratton MA, Brigande JV, et al. In vivo delivery of recombinant viruses to the fetal murine cochlea: transduction characteristics and long-term effects on auditory function. Mol Ther $2006 ; 14: 328-35$

8. Jero J, Mhatre AN, Tseng CJ, et al. Cochlear gene delivery through an intact round window membrane in mouse. Hum Gene Ther $2001 ; 12: 539-48$.

9. Wareing M, Mhatre AN, Pettis $R$, et al. Cationic liposome mediated transgene expression in the guinea pig cochlea. Hear Res $1999 ; 128: 61-9$.

10. Staecker H, Praetorius M, Baker K, Brough DE. Vestibular hair cell regeneration and restoration of balance function induced by mathl gene transfer. Otol Neurotol $2007 ; 28: 223-31$.

11. Husseman J, Raphael Y. Gene therapy in the inner ear using adenovirus vectors. Adv Otorhinolaryngol $2009 ; 66: 37-51$.

12. Kilpatrick LA, Li $Q$, Yang J, et al. Adeno-associated virus-mediated gene delivery into the scala media of the normal and deafened adult mouse ear. Gene Ther $2011 ; 18: 569-78$.

13. Liu Y, Okada T, Sheykholeslami K, et al. Specific and efficient transduction of cochlear inner hair cells with recombinant adeno-associated virus type 3 vector. Mol Ther $2005 ; 12: 725-33$.

14. Konishi $M$, Kawamoto K, Izumikawa $M$, et al. Gene transfer into guinea pig cochlea using adenoassociated virus vectors. J Gene Med $2008 ; 10: 610-8$

15. Chen X, Frisina RD, Bowers WJ, et al. HSV amplicon-mediated neurotrophin- 3 expression protects murine spiral ganglion neurons from cisplatin-induced damage. Mol Ther $2001 ; 3: 958-63$.

16. Derby ML, Sena-Esteves M, Breakefield XO, Corey DP. Gene transfer into the mammalian inner ear using HSV-1 and vaccinia virus vectors. Hear Res $1999 ; 134: 1-8$.

17. Schnepp $B C$, Jensen $R L$, Chen $C L$, et al. Characterization of adeno-associated virus genomes isolated from human tissues. J Virol $2005 ; 79$ : 14793-803

18. Gao G, Lu Y, Calcedo R, et al. Biology of AAV serotype vectors in liver-directed gene transfer to nonhuman primates. Mol Ther 2006; $13: 77-87$

19. Dong JY, Fan PD, Frizzell RA. Quantitative analysis of the packaging capacity of recombinant adeno-associated virus. Hum Gene Ther 1996 ; $7: 2101-12$

20. Lai $Y$, Yue $Y$, Liu M, et al. Efficient in vivo gene expression by trans-splicing adeno-associated viral vectors. Nat Biotechnol $2005 ; 23: 1435-9$.
21. Buning H, Perabo L, Coutelle 0 , et al. Recent developments in adenoassociated virus vector technology. J Gene Med 2008 ; $10: 717-33$.

22. Wang H, Murphy R, Taaffe D, et al. Efficient cochlear gene transfection in guinea-pigs with adeno-associated viral vectors by partial digestion of round window membrane. Gene Ther $2012 ; 19: 255-63$.

23. Shibata SB, Di Pasquale G, Cortez SR, et al. Gene transfer using bovine adeno-associated virus in the guinea pig cochlea. Gene Ther $2009 ; 16$ $990-7$.

24. Kelly MC, Chang $Q$, Pan A, et al. Atohl directs the formation of sensory mosaics and induces cell proliferation in the postnatal mammalian cochlea in vivo. I Neurosci $2012 ; 32: 6699-710$.

25. Ambros V. The functions of animal microRNAs. Nature $2004 ; 431: 350-5$

26. Vickers $T A, K o o S$, Bennett $C F$, et al. Efficient reduction of target RNAs by small interfering RNA and RNase $\mathrm{H}$-dependent antisense agents. A comparative analysis. J Biol Chem $2003 ; 278$ : 7108-18.

27. Maeda $Y$, Fukushima K, Nishizaki $K$, Smith RJ. In vitro and in vivo suppression of GJB2 expression by RNA interference. Hum Mol Genet 2005 ; 14 : 1641-50.

28. Nakaizumi T, Kawamoto K, Minoda R, Raphael Y. Adenovirus-mediated expression of brain-derived neurotrophic factor protects spiral ganglion neurons from ototoxic damage. Audiol Neurootol $2004 ; 9$ : 135-43.

29. Ahmad S, Tang W, Chang 0 , et al. Restoration of connexin 26 protein level in the cochlea completely rescues hearing in a mouse model of human connexin30-linked deafness. Proc Natl Acad Sci USA 2007 ; 104 : 1337-41.

30. Akil 0, Seal RP, Burke K, et al. Restoration of hearing in the VGLUT3 knockout mouse using virally mediated gene therapy. Neuron $2012 ; 75: 283-93$.

31. El-Amraoui A, Petit C. Thérapie cellulaire dans l'oreille interne. Med Sci (Paris) $2010 ; 26: 981-5$

32. Legendre K, Petit C, દl-Amraoui A. La cellule ciliée externe de la cochlée des mammifères. Med Sci (Paris) 2009 ; 25 : 117-20.

\section{STRESS, TRAUMATISMES ET INSOMNIES}

\section{de Jean-Pierre FRESCO}

TIRÉS À PART

S. Safieddine

\section{L’insomnie c'est}

1 Français sur 5

1 personne âgée sur 3

qui en souffrent

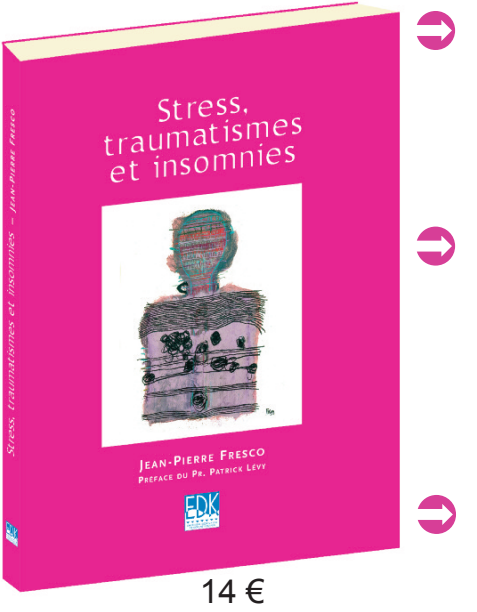

ISBN : 978-2-8425-4175-0
Une étude

sur les liens entre stress, traumatismes et insomnies.

\section{Une réflexion philosophique et sociétale sur les conditions de travail et de vie d'aujourd'hui.}

\section{Une analyse d'un mal fréquent mais encore peu compris.}

